\title{
A new method for the synthesis of chromium(IV) oxide at ambient pressure $\dagger$
}

\author{
K. Ramesha and J. Gopalakrishnan* \\ Solid State and Structural Chemistry Unit, Indian Institute of Science, Bangalore 560 012, India. \\ E-mail: gopal@sscu.iisc.ernet.in
}

Received (in Cambridge, UK) 13th April 1999, Accepted 18th May 1999

The reaction $\mathrm{CrO}_{3}+2 \mathrm{NH}_{4} \mathrm{X} \rightarrow \mathrm{CrO}_{2}+2 \mathrm{NH}_{3}+\mathrm{H}_{2} \mathrm{O}+\mathrm{X}_{2}$ $(\mathrm{X}=\mathrm{Br}, \mathrm{I})$, which occurs quantitatively at $120-150{ }^{\circ} \mathrm{C}$, provides a convenient method for the synthesis of $\mathrm{CrO}_{2}$; unlike conventional methods, the method reported here does not require the use of high pressure for the synthesis of this technologically important material.

Chromium(Iv) oxide, $\mathrm{CrO}_{2}$, is unique among the rutile-type transition metal dioxides, ${ }^{1}$ exhibiting ferromagnetic $\left(T_{\mathrm{c}}=395\right.$ $\mathrm{K})$ and metallic properties. ${ }^{2}$ Because of these properties, the material finds application as a magnetic recording medium in the form of tapes. In recent years, there has been a renewed interest in this material in view of the realization ${ }^{3}$ that it is a 'half-metallic' ferromagnet [i.e., the majority (spin-up) electrons are delocalized, while the minority (spin-down) electrons are localized, having a semiconductor-like gap in the densityof-states] with a nearly $100 \%$ spin polarization ${ }^{4}$ at the Fermi energy. An important consequence of the half-metallic nature is that films of $\mathrm{CrO}_{2}$ exhibit a low-field tunneling-type magnetoresistance $^{5}$ that appears promising for application.

Since $\mathrm{CrO}_{2}$ is a metastable phase ${ }^{2}$ (decomposing at $>288^{\circ} \mathrm{C}$ at $1 \mathrm{~atm}$ pressure of $\mathrm{O}_{2}$ ), all the methods reported for its synthesis require high pressures. ${ }^{2}$ Hydrothermal decomposition of aqueous solutions of $\mathrm{CrO}_{3}$ at elevated temperatures and pressures (typically $480{ }^{\circ} \mathrm{C}$ and $2 \mathrm{kbar}$ ) is a common method for the synthesis of polycrystalline $\mathrm{CrO}_{2}$ powders. ${ }^{2}$ Growth of single crystals ${ }^{6}$ however require much higher temperatures $\left(900-1300{ }^{\circ} \mathrm{C}\right)$ and pressures (60-65 kbar).

Considering the technological and scientific importance of $\mathrm{CrO}_{2}$, we developed a new method for its synthesis at ambient pressure. Our method is based on the low-temperature (120-150 ${ }^{\circ} \mathrm{C}$ ) reduction of $\mathrm{CrO}_{3}$ by $\mathrm{NH}_{4} \mathrm{I} / \mathrm{NH}_{4} \mathrm{Br}$ followed by annealing the solid product at temperatures below the decomposition point of $\mathrm{CrO}_{2}$. The method crucially depends on the solid state reaction given by eqn. (1)

$$
\mathrm{CrO}_{3}+2 \mathrm{NH}_{4} \mathrm{X} \rightarrow \mathrm{CrO}_{2}+2 \mathrm{NH}_{3}+\mathrm{X}_{2}+\mathrm{H}_{2} \mathrm{O}
$$

which occurs quantitatively between 120 and $150{ }^{\circ} \mathrm{C}$. Here, we describe the details of synthesis of crystalline $\mathrm{CrO}_{2}$ by this method and its characterization.

Stoichiometric quantities of $\mathrm{CrO}_{3}(20 \mathrm{mmol})$ and $\mathrm{NH}_{4} \mathrm{X}(\mathrm{X}=$ $\mathrm{Br}, \mathrm{I})(40 \mathrm{mmol})$ corresponding to eqn. (1) were thoroughly mixed under $\mathrm{CCl}_{4}$. The mixture, taken in a Pyrex glass tube connected to a vacuum line, was heated slowly in vacuo (ca. $10^{-5}$ Torr) under continuous pumping conditions as described earlier. ${ }^{7}$ Copious evolution of $\mathrm{I}_{2} / \mathrm{Br}_{2}$ occurred around $120{ }^{\circ} \mathrm{C}$. The temperature was raised to $150^{\circ} \mathrm{C}$ and held at this value until the evolution of $\mathrm{I}_{2} / \mathrm{Br}_{2}$ ceased $(2-3 \mathrm{~h})$. The liberated $\mathrm{I}_{2} / \mathrm{Br}_{2}$ condensed in a liquid nitrogen trap provided a quantitative measure of the extent of the reduction. ${ }^{7}$ Significantly, reaction (1) does not occur with $\mathrm{NH}_{4} \mathrm{Cl}$. This is consistent with the redox characteristics of $\mathrm{Cr}^{\mathrm{VI}}$ which oxidizes $\mathrm{I}^{-}$and $\mathrm{Br}-$ but not $\mathrm{Cl}^{-}$ in aqueous solutions. ${ }^{8}$

The black solid product of reaction (1) was stable in air; it was however non-magnetic and amorphous to X-rays [Fig. 1(a)]. Pellets of this product were annealed in evacuated sealed

$\dagger$ Contribution No. 1412 from the Solid State and Structural Chemistry Unit. glass tubes at various temperatures and times. We found that annealing at $195 \pm 5{ }^{\circ} \mathrm{C}$ for one week yielded crystalline $\mathrm{CrO}_{2}$ with rutile structure. The lattice parameters, $a=4.419$ (1), $c=$ 2.915 (1) $\AA$, derived from the X-ray powder diffraction pattern [Fig. 1(b)] are in excellent agreement with the values reported for $\mathrm{CrO}_{2}$ in the literature. ${ }^{9}$ Determination of oxygen content by iodometric titration revealed that the product is stoichiometric $\left(\mathrm{CrO}_{1.99 \pm 0.02}\right)$.

Measurement of electrical resistivity and magnetic susceptibility revealed that the $\mathrm{CrO}_{2}$ prepared here is metallic and ferromagnetic as expected. The resistivity and susceptibility data of our samples (Fig. 2) are comparable to the corresponding data reported in the literature ${ }^{5}$ for bulk polycrystalline samples

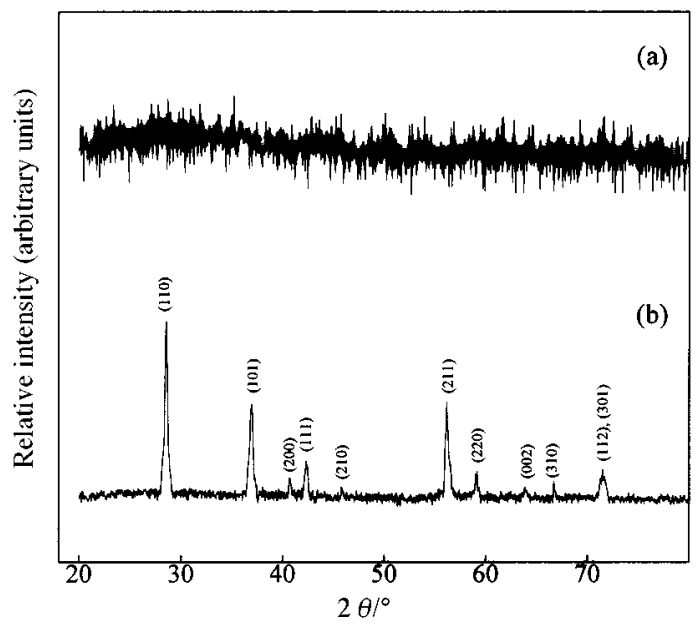

Fig. 1 Powder XRD patterns $(\mathrm{Cu}-\mathrm{K} \alpha)$ of (a) solid product of reaction (1) with $\mathrm{NH}_{4} \mathrm{I}$ and (b) product (a) annealed at $195 \pm 5{ }^{\circ} \mathrm{C}$ for one week corresponding to crystalline $\mathrm{CrO}_{2}$.

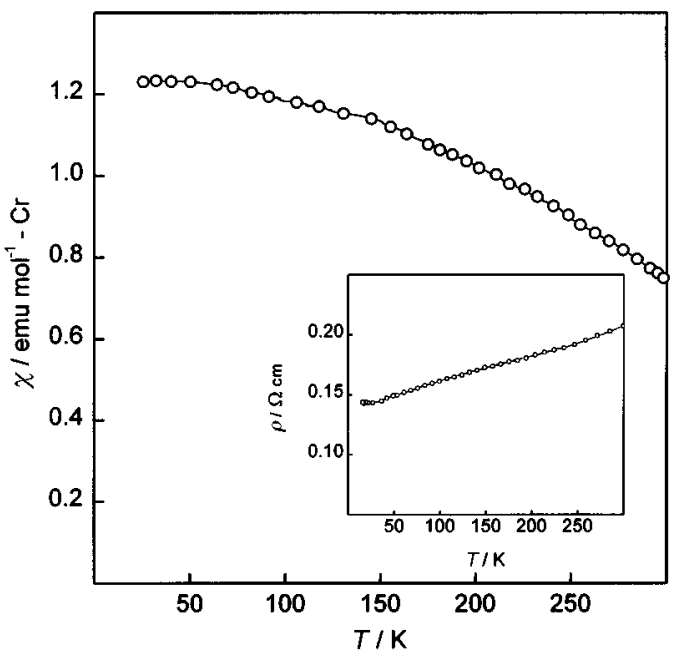

Fig. 2 Temperature dependence of magnetic susceptibility of $\mathrm{CrO}_{2}$ measured at $0.5 \mathrm{~T}$. Inset shows the temperature dependence of electrical resistivity of the same sample. 


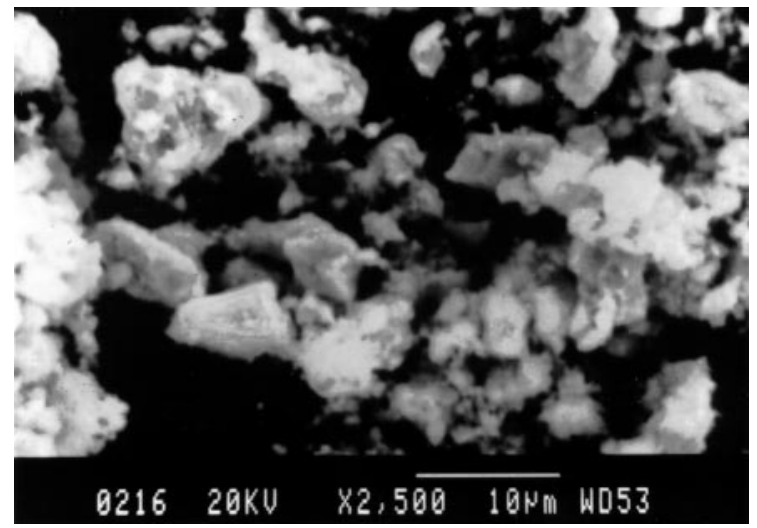

Fig. 3 SEM image of crystalline $\mathrm{CrO}_{2}$.

as well as films of $\mathrm{CrO}_{2}$. An SEM study showed that the samples are fairly crystalline, the particle sizes being in the range of a few $\mu \mathrm{m}$ (Fig. 3).

We believe that the key to the success of the method lies in the quantitative nature of reaction (1) which can be controlled to give an average oxidation state of $\mathrm{Cr}^{\mathrm{IV}}$ in the solid state by using the required quantity of $\mathrm{NH}_{4} \mathrm{I} / \mathrm{NH}_{4} \mathrm{Br}$ in the reaction mixture. This is in contrast to reactions between $\mathrm{CrVI}^{\mathrm{VI}}$ and $\mathrm{I}^{-} / \mathrm{Br}^{-}$in aqueous media, which proceed directly to give $\mathrm{Cr}^{\mathrm{III}}$; i.e. $\mathrm{Cr}^{\mathrm{VI}}$ is not accessible as a stable species in aqueous media by redox reactions. An investigation of the $\mathrm{X}$-ray morphous $\mathrm{CrO}_{2}$ would throw light on the mechanism of reaction (1) which stabilizes $\mathrm{Cr}^{\mathrm{VI}}$ in the solid state.
We can also prepare rutile-related (monoclinic) $\mathrm{VO}_{2}$ as well as solid solutions of $\mathrm{Cr}_{1-x} \mathrm{~V}_{x} \mathrm{O}_{2}$ by the same method starting from $\mathrm{V}_{2} \mathrm{O}_{5}$ and $\mathrm{V}_{2} \mathrm{O}_{5}-\mathrm{CrO}_{3}$ mixtures. Therefore, we believe that the method described here can be extended to the synthesis of other metastable oxides containing $\mathrm{Cr}^{\mathrm{IV}}$ and/or $\mathrm{V}^{\mathrm{IV}}$, in general.

We thank the Department of Science and Technology, Government of India (Project No. SP/S1/H-17/97) for financial support. K. R. thanks the Council of Scientific and Industiral Research, New Delhi for the award of a fellowship.

\section{Notes and references}

1 D. B. Rogers, R. D. Shannon, A. W. Sleight and J. L. Gillson, Inorg. Chem., 1969, 8, 841 .

2 B. L. Chamberland, CRC Crit. Rev. Solid State Mater. Sci., 1977, 7, 1.

3 K. P. Kämper, W. Schmitt, G. Güntherodt, R. J. Gambino and R. Ruf, Phys. Rev. Lett., 1987, 59, 2788.

4 R. J. Soulen Jr., J. M. Byers, M. S. Osofsky, B. Nadgorny, T. Ambrose, S. F. Cheng, P. R. Broussard, C. T. Tanaka, J. Nowak, J. S. Moodera, A. Barry and J. M. D. Coey, Science, 1998, 282, 85.

5 H. Y. Hwang and S.-W. Cheong, Science, 1997, 278, 1607.

6 B. L. Chamberland, Mater. Res. Bull., 1967, 2, 827.

7 V. Bhat and J. Gopalakrishnan, J. Chem. Soc. Chem. Commun., 1986, 1644.

8 J. Bassett, R. C. Denney, G. H. Jeffery and J. Mendham, Vogel's Textbook of Quantitative Inorganic Analysis, Longman, London, 4th edn., 1978, p. 51.

9 JCPDS-International Centre for Diffraction Data Card No. 43-1040.

Communication $9 / 02927 C$ 